\title{
Evolución de la mortalidad por enfermedad isquémica del corazón e infarto agudo del miocardio en Costa Rica, 1970-2001
}

\author{
Marlene Roselló Araya ${ }^{1}$ y Sonia Guzmán Padilla ${ }^{1}$
}

Forma de citar

Roselló Araya M, Guzmán Padilla S. Evolución de la mortalidad por enfermedad isquémica del corazón e infarto agudo del miocardio en Costa Rica, 1970-2001. Rev Panam Salud Publica. 2004;16(5): 295-301.

RESUMEN Objetivo. Describir la evolución epidemiológica de la mortalidad por enfermedades cardiovasculares (ECV), enfermedad isquémica del corazón (EIC) e infarto agudo del miocardio (IAM) en Costa Rica, según el sexo y la región geográfica, entre los años 1970 y 2001.

Métodos. Se realizó un estudio descriptivo de la mortalidad por ECV, EIC e IAM en Costa Rica entre 1970 y 2001. La información se obtuvo de la base de datos del Centro Centroamericano de Población. Los datos de mortalidad por EIC e IAM entre 1970 y 2001 se analizaron según la revisión mas actualizada de la Clasificación Internacional de Enfermedades (CIE). El territorio costarricense se dividió en: región metropolitana (8 cantones), región semiurbana Valle Central (18 cantones), región rural Valle Central (17 cantones), región semiurbana bajura (12 cantones) y región rural bajura (26 cantones). La tendencia de la mortalidad se analizó por quinquenios (entre 1970 y 1999) y el bienio de 2000-2001 mediante las tasas brutas de mortalidad por 100000 habitantes para cada causa o grupo de causas, según la edad, el sexo y el año de fallecimiento. Las tasas se ajustaron por edad, sexo, año de fallecimiento y región geográfica mediante el método directo, usando como población estándar la de América Latina en 1960.

Resultados. La mortalidad por ECV disminuyó $33 \%$ en promedio $(46,6 \%$ en mujeres y $20,2 \%$ en hombres), mientras que la mortalidad por EIC aumentó un promedio de 18,4\% (6,1\% mujeres y $28,4 \%$ hombres). La tasa ajustada de mortalidad por IAM en hombres aumentó $12,8 \%$ en el período de estudio, con un ligero descenso de 4,4\% en mujeres. La mortalidad por ECV, EIC e IAM fue mayor en hombres que en mujeres durante todo el período de estudio. Las tasas de mortalidad por EIC e IAM se elevaron en las regiones semiurbanas y rurales, especialmente en la región rural bajura, donde aumentaron con respecto al quinquenio de 1995-1999 en 123,9\% y 76,9\%, respectivamente.

Conclusiones. Se observó una disminución de la mortalidad por ECV. Las mayores tasas se encontraron en hombres y en personas de 75 años de edad o más. Los mayores incrementos en las tasas de mortalidad por EIC e IAM se observaron en las regiones semiurbanas y rurales del país, en hombres, y en personas de 75 años o más. Se debe prestar especial atención al aumento de los factores de riesgo de padecer ECV, como el tabaquismo, la hipertensión arterial, el sobrepeso y la obesidad, el sedentarismo y la alimentación inadecuada.

Palavras-chave Mortalidad, infarto del miocardio, enfermedades cardiovasculares, isquemia miocárdica, Costa Rica.

1 Instituto Costarricense de Investigación y Enseñanza en Nutrición y Salud (INCIENSA), San José, Costa Rica. La correspondencia debe dirigirse a: Marlene Roselló, Apdo. 4, Tres Ríos, Cartago, Costa Rica. Correo electrónico: mrosello@inciensa. sa.cr
Costa Rica sigue un modelo rápido de transición epidemiológica, al igual que los países de Europa Central y al- gunos otros países latinoamericanos, como Chile y Cuba. En la década de 1970, la principal causa de muerte en 
Costa Rica eran las enfermedades infecciosas. Sin embargo, en los últimos años las enfermedades del aparato circulatorio y el cáncer pasaron a ser las principales causas de muerte. A esto se debe sumar que la esperanza de vida en el país se elevó de 75 a 79 años, lo que aumenta la exposición a los factores de riesgo asociados con el envejecimiento, que generalmente facilitan la aparición de enfermedades crónicas degenerativas (1-4).

Las enfermedades cardiovasculares (ECV) constituyen la principal causa de muerte en Costa Rica desde 1970, tanto en hombres como en mujeres mayores de 30 años de edad. Las ECV son la causa de alrededor de $33 \%$ del total de defunciones en Costa Rica, porcentaje similar al encontrado en América Latina y el Caribe (31\%) (5). Del total de muertes por ECV, $48 \%$ corresponde a la enfermedad isquémica del corazón (EIC), 22,7\% a las enfermedades cerebrovasculares y $11 \%$ a causas asociadas con la hipertensión arterial. Del total de muertes por EIC en el año $2001,68,4 \%$ se produjeron por infarto agudo del miocardio (IAM) ${ }^{2}$.

En el presente estudio se describe la evolución epidemiológica de la mortalidad por ECV, EIC e IAM en Costa Rica, según el sexo y la región geográfica, entre los años 1970 y 2001.

\section{MATERIALES Y MÉTODOS}

Se realizó un estudio descriptivo de la mortalidad por ECV, EIC e IAM en Costa Rica entre 1970 y 2001.

La información estadística se tomó de la base de datos de mortalidad del Centro Centroamericano de Población, (6) que contiene la información generada por el Instituto Nacional de Estadística y Censos (INEC) de Costa Rica a partir de los certificados de defunción recibidos de los establecimientos de salud. Cuando un paciente muere fuera de un hospital o durante las primeras 24 horas de su ingreso en él, la morgue judicial se encarga de establecer la causa de muerte. En todos los

\footnotetext{
2 Calculado a partir de los datos del Centro Centro-
} americano de Población (6). casos, personal entrenado del INEC codifica las causas de muerte según la Clasificación Internacional de Enfermedades (CIE) más actualizada. En 1991, la Organización Panamericana de la Salud (OPS) clasificó a Costa Rica entre los países con menor subregistro en sus estadísticas de mortalidad (7). El subregistro de defunciones en el año 1970 se calculó en $8,1 \%$, mientras que en el año 2000 este fue de solo $0,3 \%$ (6).

Las variables analizadas fueron la edad, el sexo y la región geográfica. Los datos de 1970 a 1979 se analizaron según la octava revisión de la CIE (8) de la Organización Mundial de la Salud (OMS). De 1980 a 1996 se utilizó la novena revisión (9) y para el período de 1997 a 2001 se empleó la décima edición (10). Los códigos de las enfermedades utilizadas en la octava y novena revisiones de la CIE fueron los de enfermedad isquémica del corazón (CIE 410-414) e infarto agudo del miocardio (CIE 410). En la décima revisión se utilizaron los códigos de enfermedad isquémica (CIE I20-I25) e infarto agudo del miocardio (CIE I21).

Según el Centro Centroamericano de Población, Costa Rica se divide en cinco regiones geográficas de acuerdo con el grado de urbanización de los cantones que las componen y de su ubicación dentro o fuera del Valle Central: región metropolitana (8 cantones), región semiurbana Valle Central (18 cantones), región rural Valle Central (17 cantones), región semiurbana bajura (12 cantones) y región rural bajura (26 cantones) (11).

La tendencia de la mortalidad se analizó por quinquenios (entre $1970 \mathrm{y}$ 1999) y el bienio 2000-2001 mediante las tasas brutas de mortalidad por 100000 habitantes para cada causa o grupo de causas según la edad, el sexo y el año de fallecimiento. Dado que la estructura por edad y sexo de la población es un factor determinante de la mortalidad, se ajustaron las tasas por el método directo (12) usando como estándar la población de América Latina en 1960 (13).

Las tasas de mortalidad se analizaron mediante estadísticas descriptivos con intervalos de confianza de 95\% (IC95\%). Para comparar los resultados de ECV con la información de otras regiones y países se emplearon los datos del grupo de edad entre los 35 y 74 años. Se utilizó un nivel de significación estadística de 0,05.

\section{RESULTADOS}

En el período estudiado, la tasa ajustada de mortalidad por ECV en personas entre los 35 y 74 años de edad en Costa Rica mostró una tendencia descendente entre el quinquenio de 19701974 (59,5 por 100000 habitantes; IC95\%: 56,9 a 62,1$)$ y el quinquenio de 1995-1999 (40,6 por 100000 habitantes; IC95\%: 39,4 a 41,8$)$ y se mantuvo baja en el bienio de 2000-2001 (figura 1). La tasa de mortalidad también disminuyó entre los quinquenios de 1970-1974 y 1995-1999, tanto en hombres $(63,1$ por 100000 habitantes [IC95\%: 59,4 a 66,8] frente a 50,3 por 100000 habitantes [IC95\%: 48,4 a 52,3], respectivamente) como en mujeres (55,3 por 100000 habitantes [IC95\%: 52,0 a 58,6] frente a 31,2 por 100000 habitantes [IC95\%: 29,9 a 32,6], respectivamente). En ambos casos la tasa para el bienio de 2000-2001 se mantuvo estable. La menor tasa de mortalidad registrada en el período estudiado se observó en mujeres durante el quinquenio de 1995-1999 (figura 1).

En el primer quinquenio de la década de 1970 se observó un descenso abrupto de la mortalidad por ECV en ambos sexos. Este continuó, aunque de manera menos pronunciada, en la década de 1990. En su conjunto, la reducción de la mortalidad por ECV durante los años estudiados fue de $46,6 \%$ en mujeres y de $20,2 \%$ en hombres, equivalente a un promedio de $33,0 \%$ (cuadro 1 ).

Durante el período analizado, la tasa de mortalidad total por EIC aumentó un promedio de $18,4 \%(7,0 \%$ en mujeres y $28,4 \%$ en hombres; $P<0,05$ ). No obstante, no se observó una tendencia estable en ninguno de los dos sexos (figura 2).

La tasa ajustada de mortalidad por IAM en hombres aumentó $12,8 \%$ entre el quinquenio de 1970-1974 (40,0 por 100000 habitantes; IC95\%: 35,3 a 44,8) y 
FIGURA 1. Tasas ajustadas de mortalidad por enfermedades cardiovasculares en personas de 35 a 74 años, según el sexo. Costa Rica, 1970-2001

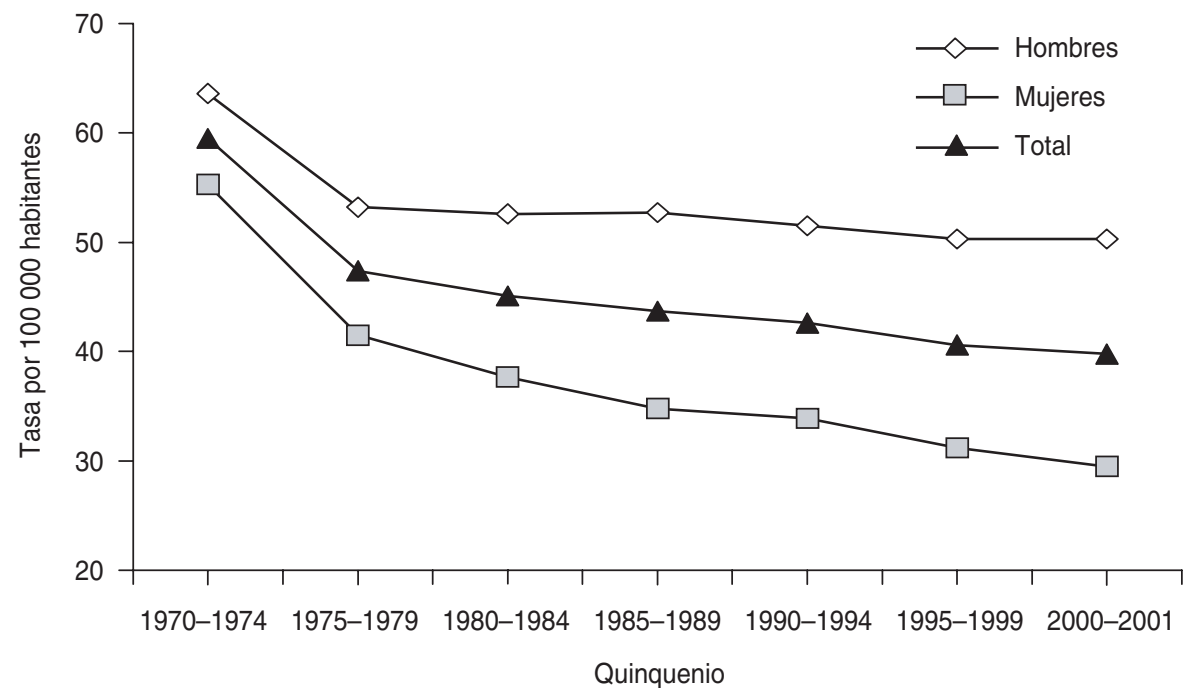

Fuente: http://www.censos.ccp.ucr.ac

CUADRO 1. Variación porcentual quinquenal de las tasas estandarizadas de mortalidad por enfermedades cardiovasculares en la población de 35 a 74 años de edad. Costa Rica, 1970-2001

\begin{tabular}{lrcr}
\hline & \multicolumn{3}{c}{ Modificación porcentual } \\
\cline { 2 - 4 } Intervalos comparados & Hombres & Mujeres & Total \\
\hline $1975-1979$ / 1970-1974 & $-15,6$ & $-24,6$ & $-20,3$ \\
$1980-1984$ / 1975-1979 & $-1,1$ & $-9,1$ & $-4,8$ \\
$1985-1989$ / 1980-1984 & 0 & $-8,3$ & $-3,1$ \\
$1990-1994$ / 1985-1989 & $-2,3$ & $-2,5$ & $-2,5$ \\
$1995-1999$ / 1990-1994 & $-2,3$ & $-7,9$ & $-4,9$ \\
$2000-2001$ / 1995-1999 & 0 & $-5,4$ & $-1,9$ \\
$1995-1999$ / 1970-1974 & $-20,2$ & $-43,6$ & $-31,8$ \\
$2000-2001 /$ 1970-1974 & $-20,2$ & $-46,6$ & $-33,0$ \\
\hline
\end{tabular}

Fuente: http://www.censos.ccp.ucr.ac

el bienio de 2000-2001 (45,1 por 100000 habitantes; IC95\%: 42,3 a 48). La tasa de mortalidad en mujeres mostró un ligero descenso de $4,4 \%$ entre ambos quinquenios $(27,2$ por 100000 habitantes [IC95\%: 23,5 a 30,9] frente a 26,0 por 100000 habitantes [IC95\%: 24,9 a 26,9], respectivamente) (figura 2).

Durante todo el período de estudio, la mortalidad por ECV, EIC e IAM tanto en tasas brutas como en cifras absolutas- fue mayor en hombres que en mujeres, incluso después de hacer ajustes según la edad. La razón entre las tasas de mortalidad por IAM en hombres y en mujeres aumentó hasta alcanzar su valor máximo $(1,89)$ en el quinquenio de 1985-1989. A partir de la década de 1990, la mortalidad por IAM en hombres disminuyó, lo que redujo la brecha entre las tasas de mortalidad por esa causa en hombres y en mujeres (cuadro 2).

En lo referente a la EIC y al IAM, las tasas de mortalidad masculina en casi todos los grupos de edad duplican las tasas observadas en mujeres (datos no mostrados). En el caso de la EIC, la tasa fue más alta en el grupo de personas de 75 años de edad o más en ambos sexos, mientras que en el del IAM, los hombres de 35 a 44 años de edad presentaron las mayores tasas de mortalidad hasta el quinquenio de 1990-1994.

Con respecto a la edad de defunción, en todo el período de estudio se observó una diferencia promedio de dos años entre los hombres y las mujeres, tanto por EIC $(69,8$ frente a 72,9 años) como por IAM (69,0 frente a 71,6 años) (datos no mostrados).

Durante los primeros 20 años de estudio, las tasas de mortalidad por EIC e IAM fueron más elevadas en la región metropolitana. Sin embargo, en el último quinquenio (1995-1999) la región semiurbana Valle Central mostró las mayores tasas de mortalidad por EIC (43,1 por 100000 habitantes). Es importante resaltar que en el bienio de 2000-2001, las tasas se elevaron en las regiones semiurbana y rural de la bajura, tanto para la mortalidad por EIC como por IAM (cuadro 3), especialmente en la región de la rural bajura, donde los porcentajes aumentaron con respecto al quinquenio de 1995-1999 en 123,9 y 76,9 , respectivamente.

\section{DISCUSIÓN}

En Costa Rica, las ECV ocasionan la tercera parte de las defunciones y constituyen la primera causa de muerte en adultos. No obstante, la tasa de mortalidad por estas enfermedades se redujo un promedio de $33 \%$ en el período de estudio. En países de diferentes regiones se ha observado una reducción de $39 \%$ en hombres y de $36 \%$ en mujeres $(14,15)$, pero en Costa Rica este descenso ha sido mucho mayor en mujeres $(46,6 \%)$ que en hombres $(20,2 \%)$.

A la EIC le correspondió cerca del $50 \%$ de las muertes por ECV en Costa Rica durante prácticamente todo el período de estudio, similar a lo informado en diversos países $(15,16)$. Sin embargo, a diferencia del descenso observado a finales de la década de 1970 en los Estados Unidos y Canadá y a lo largo de esa década en la mayor parte de los países de Europa Occidental, la 
FIGURA 2. Tasas ajustadas de mortalidad por enfermedad isquémica del corazón (EIC) e infarto agudo del miocardio (IAM), según el sexo. Costa Rica, 1970-2001
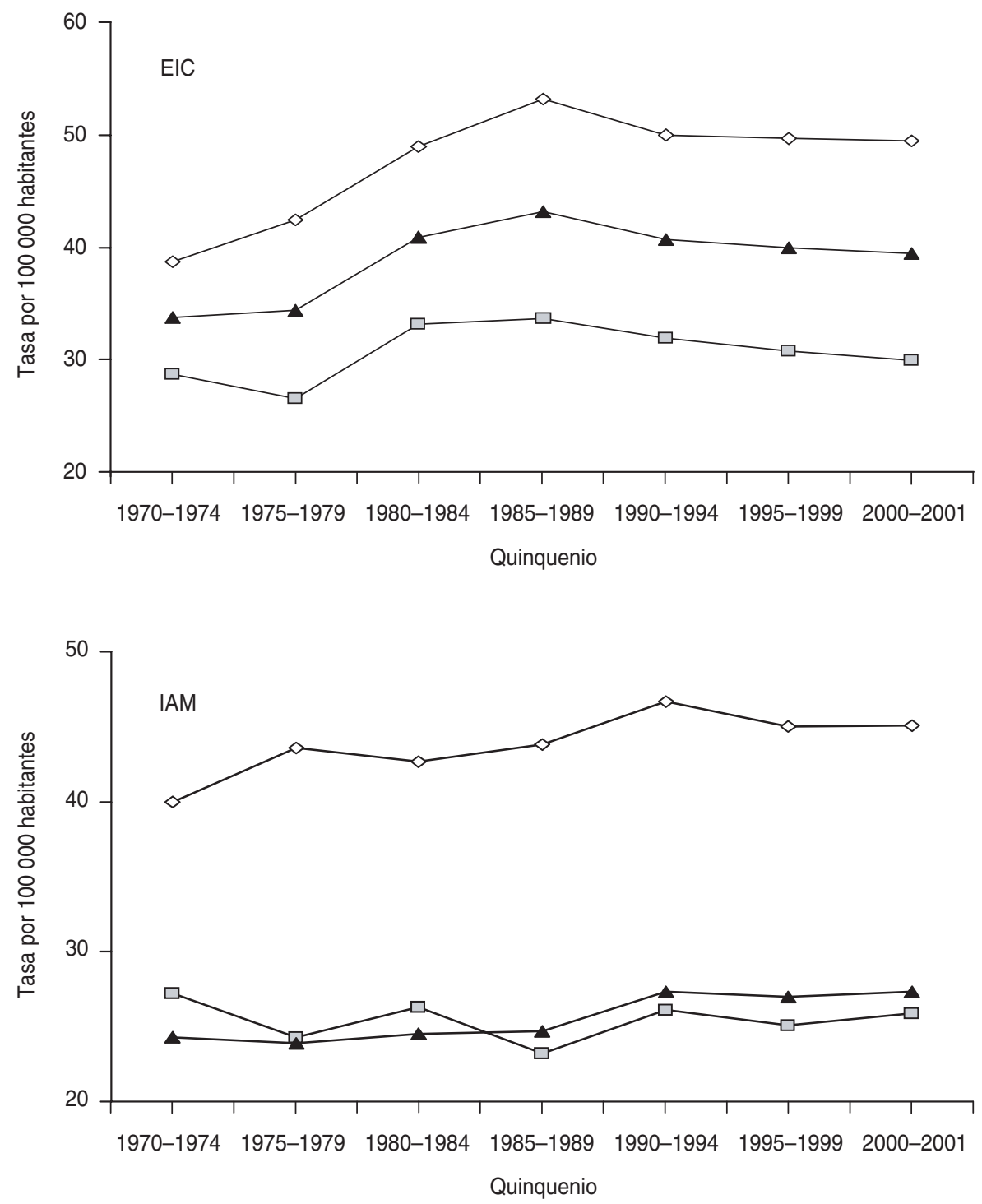

$\checkmark$ Hombres $\square-$ Mujeres $\longrightarrow$ Total

Fuente: http://www.censos.ccp.ucr.ac

tasa de mortalidad por EIC aumentó en Costa Rica tanto en hombres como en mujeres (14).

Al igual que ocurre en otros países, las mayores tasas de mortalidad, tanto por EIC como por ECV, se observaron en personas de 75 años de edad o más. Las tasas de mortalidad por ECV aumentaron con la edad, independiente- mente del sexo, lo que puede explicarse, en parte, por su asociación con la enfermedad aterosclerótica y con los numerosos factores de riesgo vinculados con el envejecimiento $(17,18)$. Las edades promedio de muerte por ECV, EIC e IAM en Costa Rica (70,7 años, 71,6 años y 69,8 años, respectivamente) fueron similares a las informadas para
CUADRO 2. Razón hombre:mujer de las tasas de mortalidad por enfermedades cardiovasculares (ECV), enfermedad isquémica del corazón (EIC) e infarto agudo del miocardio (IAM) en la población de 35 a 74 años de edad. Costa Rica, 1970-2001

\begin{tabular}{cccc}
\hline Período & ECV & EIC & IAM \\
\hline $1970-1974$ & 1,14 & 1,34 & 1,47 \\
$1975-1979$ & 1,28 & 1,60 & 1,79 \\
$1980-1984$ & 1,39 & 1,48 & 1,62 \\
$1985-1989$ & 1,51 & 1,58 & 1,89 \\
$1990-1994$ & 1,51 & 1,57 & 1,79 \\
$1995-1999$ & 1,61 & 1,62 & 1,73 \\
$2000-2001$ & 1,70 & 1,65 & 1,74 \\
\hline
\end{tabular}

Fuente: http://www.censos.ccp.ucr.ac

algunos países de América del Norte, América del Sur y el Caribe ${ }^{3}$.

Los hombres que murieron por EIC o por IAM eran en promedio dos años más jóvenes que las mujeres, algo similar a lo que ocurre en Cuba, pero muy diferente de lo observado en otros países de la Región, en los que esta diferencia es de tres a seis años ${ }^{3}$. Desde hace 10 años, la población costarricense transita por un proceso de envejecimiento relativo que continuará en los próximos años. En 2003, el grupo de edad de 45 a 64 años constituía el 15,3\% de la población y la esperanza de vida al nacer era de 78,5 años (76,2 años para los hombres y 81,0 para las mujeres) (19).

En el período estudiado, las tasas de mortalidad por ECV y EIC en hombres fueron siempre mayores que las observadas en mujeres, patrón similar al de otros países de América del Norte, América del Sur y el Caribe (7). A pesar de que en algunos países como España (16) - la razón de las tasas de mortalidad hombre:mujer descendió, en Costa Rica se observó un aumento progresivo de esa razón en la mortalidad por ECV, EIC e IAM (cuadro 2), lo que refleja una mayor mortalidad en los hombres. Las razones observadas fueron similares a las encontradas en algunos países europeos como Grecia $(1,3)$ y Finlandia $(1,8)$,

\footnotetext{
3 Calculado a partir de los datos del Centro Centroamericano de Población (6).
} 
CUADRO 3. Tasas ajustadas de mortalidad por enfermedad isquémica del corazón e infarto agudo del miocardio por 100000 habitantes, según la región geográfica. Costa Rica, 1970-2001

\begin{tabular}{|c|c|c|c|c|c|c|c|c|c|c|}
\hline $\begin{array}{l}\text { Período } \\
\text { analizado }\end{array}$ & \multicolumn{5}{|c|}{ Enfermedad isquémica del corazón } & \multicolumn{5}{|c|}{ Infarto agudo del miocardio } \\
\hline 1970-1974 & 45,4 & 40,1 & 29,6 & 31,2 & 14,6 & 31,7 & 26,7 & 22,9 & 24,3 & 11,6 \\
\hline $1975-1979$ & 41,2 & 39,0 & 32,4 & 32,8 & 19,5 & 27,5 & 27,4 & 26,7 & 24,8 & 14,5 \\
\hline $1980-1984$ & 50,0 & 45,6 & 38,7 & 36,7 & 24,8 & 29,1 & 27,3 & 27,9 & 24,9 & 14,9 \\
\hline 1995-1999 & 42,8 & 43,1 & 38,4 & 38,2 & 32,7 & 31,7 & 31,3 & 29,5 & 25,0 & 20,5 \\
\hline 2000-2001 & 47,0 & 43,1 & 35,4 & 34,2 & 33,9 & 32,0 & 29,9 & 27,4 & 22,3 & 23,1 \\
\hline $\begin{array}{r}\text { Variación entre } \\
1995-1999 \text { y }\end{array}$ & & & & & & & & & & \\
\hline $1970-1974(\%)$ & $-5,7$ & 7,5 & 29,7 & 22,4 & 123,9 & 0 & 17,2 & 28,8 & 2,8 & 76,7 \\
\hline
\end{tabular}

Fuente: http://www.censos.ccp.ucr.ac

aunque fueron muy inferiores a las encontradas en otros países desarrollados, donde esta razón fue de 2,5 a 4,5 (20-22). Esta mayor mortalidad masculina puede deberse, en parte, a algunos factores de riesgo, como el tabaquismo y el consumo de bebidas alcohólicas, que son más frecuentes en los hombres.

La mortalidad por EIC en la población urbana fue significativamente mayor que la encontrada en las zonas rurales. Sin embargo, se observó una tendencia ascendente en la mortalidad por EIC en las regiones rurales, posiblemente debido a la adopción de hábitos y estilos de vida semejantes a los de la población urbana. Este proceso también ha sido documentado en España y en algunas ciudades de los Estados Unidos, lo que pudiera corroborar que, además de determinantes genéticos, el grado de urbanización y la condición socioeconómica pueden incidir en el patrón de mortalidad de un país (23-25).

El consumo de bebidas alcohólicas está asociado con la mortalidad por ECV. Según el Instituto sobre Alcoholismo y Farmacodependencia (IAFA), la prevalencia del consumo de alcohol en Costa Rica en 1995 fue de 62,3\%, con una incidencia ese año de 120 por 1000 habitantes, cifra superior a la encontrada en 1990 (116 por 1000 habitantes) $(26,27)$. La encuesta basal realizada en 2000 como parte del Conjunto de Acciones para la Reducción Multifactorial de Enfermedades No Transmisibles (CARMEN) determinó que 16,4\% (IC95\%: 13,7 a 19,1) de los hombres y 2,8\% (IC95\%: 1,7 a 3,9) de las mujeres habían consumido 30 ó más bebidas alcohólicas en el último mes y habían tomado 5 ó más tragos seguidos (28).

Otro factor de riesgo asociado con la mortalidad por ECV, EIC e IAM es el tabaquismo. En el mundo, la prevalencia de tabaquismo en 1998 fue de 33\% y se calcula que la prevalencia de enfermedades asociadas con este hábito, entre ellas las ECV, aumentará de 2,6\% en 1990 a 9\% en el año 2020 (14). En Costa Rica, la incidencia de tabaquismo en la población de 12 a 70 años aumentó de 14 por 1000 habitantes en 1990 a 22 por 1000 habitantes en 1995 $(26,27)$. De los fumadores, $17 \%$ se ubicaron en la categoría de "fumador activo" y de ellos, 81,1\% eran hombres. La encuesta CARMEN encontró que la prevalencia de consumo de tabaco fue de $23,7 \%$ en los hombres y de $10 \%$ en las mujeres (28).

El sedentarismo también incide en el aumento de la prevalencia de ECV. Según el IAFA, solo $22,3 \%$ de la población costarricense realiza ejercicio físico. El grupo de 15 a 24 años de edad realiza ejercicio físico con mayor regularidad, pero esta actividad disminuye a medida que aumenta la edad $(26,27)$.
Otro factor que influye en la mortalidad por ECV es el peso corporal. Las encuestas nacionales de nutrición han encontrado una tendencia a la obesidad en la población costarricense. En 1996, la prevalencia de sobrepeso en mujeres entre 20 y 44 años de edad era de $45,9 \%$ (11,3\% más que la encontrada en 1982) y esa prevalencia se elevó a $75 \%$ en mujeres de 45 a 59 años (29). Por otra parte, $59,4 \%$ de la población que participó en la encuesta CARMEN (60,4\% de las mujeres y 58,1\% de los hombres) presentó algún grado de sobrepeso u obesidad (30). Estas cifras son similares a las encontradas en los Estados Unidos, donde cerca de $60 \%$ de la población adulta tiene sobrepeso y $30 \%$ es obesa (31).

Desde el año 1991, las autoridades nacionales de salud han informado que la dieta del costarricense no es suficientemente equilibrada, por lo que se considera un factor de riesgo aterógeno (32). El consumo de grasa en la población en general se incrementó de $20,0 \%$ en 1966 a $27,4 \%$ en 1996, y alcanza 35\% en los estratos socioeconómicos altos $(3,33)$. Según los resultados de la encuesta CARMEN, el aporte dietético de carbohidratos y proteínas es adecuado (63\% y $12 \%$, respectivamente), pero la alimentación de más de $50 \%$ de la población contiene un $28 \%$ de grasas, cifra superior a la recomendada (menos de 25\%) (33, 34). Otra característica de la dieta del cos- 
tarricense es la poca ingesta de fibra (entre 11 y 25 g en total) $(33,35)$.

Las concentraciones elevadas de colesterol en la sangre constituyen otro factor de riesgo de ECV por su asociación con las lesiones ateromatosas (36). En una investigación realizada en Costa Rica, Roselló y colaboradores encontraron que $43,3 \%$ de la población estudiada de adultos tenía más de 200 $\mathrm{mg} / \mathrm{dL}$ de colesterol en la sangre (37), mientras que según la encuesta CARMEN, ese porcentaje asciende a $57,1 \%$ (30). Además, 44,4\% de la población presentaba concentraciones séricas de triglicéridos mayores de $150 \mathrm{mg} / \mathrm{dL}$, $44 \%$ tenía valores séricos de colesterol de lipoproteínas de alta densidad (HDL) menores de $40 \mathrm{mg} / \mathrm{dL}$ y 51,2\% de los estudiados tenían valores séricos de colesterol de lipoproteínas de baja densidad (LDL) por encima de $130 \mathrm{mg} / \mathrm{dL}$ (30). Tanto para el colesterol de LDL como para los triglicéridos, la prevalencia fue similar en hombres y mujeres, pero las mujeres mostraron valores más elevados de colesterol de HDL que los hombres (30).

A estos factores de riesgo se suma una prevalencia de hipertensión arterial de $9 \%$ en mayores de 15 años de edad
(38) y de 2,8\% de diabetes notificada por el propio paciente (39). Según la encuesta CARMEN, $4 \%$ de la población costarricense padece de diabetes (30).

La investigación y la vigilancia de la situación de salud constituyen elementos indispensables para la prevención y el control de las ECV y, por ende, de la EIC y el IAM. Aún queda mucho por conocer acerca de los factores de riesgo, de su distribución en la población y de la eficacia de las intervenciones dirigidas a controlar estos problemas de salud (40).

Los resultados de este estudio concuerdan con los hallazgos epidemiológicos encontrados en la bibliografía internacional (41), en particular en relación con la estabilidad - e incluso el descenso- en las tasas de mortalidad por ECV, a pesar del aumento de su prevalencia. Esto podría explicarse, en parte, por los avances científicos que permiten diagnosticar y tratar tempranamente a las personas afectadas, por el considerable aumento en la oferta de servicios de salud y por la mayor disposición de las personas a atenderse en centros hospitalarios. Además, la elevada prevalencia de factores de riesgo cardiovascular en Costa Rica (el tabaquismo, la hipertensión arterial, las dislipidemias, la diabetes mellitus, el sobrepeso y la obesidad, el sedentarismo y la alimentación inadecuada) explica en parte el incremento observado en la mortalidad por EIC e IAM $(26,27,33,34)$.

Se sabe que es posible reducir en $23 \%$ la mortalidad por estas causas si se ponen en marcha estrategias dirigidas a reducir los factores de riesgo relacionados con las condiciones de vida, como el fomento del ejercicio físico y de una dieta equilibrada y la reducción del tabaquismo $(1,5)$. Sin embargo, para lograr esa meta es necesario aplicar un enfoque intersectorial que estimule la adopción de estilos de vida saludables. Para ello no basta con contar con la responsabilidad individual de cada miembro de la población, sino que se deben establecer redes de apoyo social y familiar con el respaldo del Estado. Entre otras medidas, se debe dotar a las comunidades de infraestructuras adecuadas para la recreación y la práctica sistemática del ejercicio físico, se debe facilitar el acceso a una dieta equilibrada y se debe regular legalmente la venta de tabaco y alcohol (42).

\section{REFERENCIAS}

1. Mosley W, Bobadilla J, Jamison D. The health transition: implications for health policy in developing countries. En: Jamison D, Mosley W, Measham A, Bobadilla J, eds. Disease control priorities in developing countries. Washington, D.C.: World Bank, Oxford University Press; 1993. Pp. 673-99.

2. Nicholls E. Diferenciales de mortalidad de las enfermedades no transmisibles según el nivel socioeconómico: el caso de América Latina. Bol Oficina Sanit Panam. 1993;115:255-69.

3. Caja Costarricense de Seguro Social. Plan de atención a la salud de las personas. San José, Costa Rica: Corporación Litográfica Internacional; 2001.

4. Omran A. The epidemiologic transition in the Americas. Washington, D.C.: Pan American Health Organization, World Health Organization, University of Maryland at College Park; 1999.

5. Organización Panamericana de la Salud. Enfermedades cardiovasculares en especial la hipertensión arterial. Washington, D.C.: OPS; 2000.

6. Centro Centroamericano de Población, Universidad de Costa Rica. Datos de mortalidad. En: Censos y grandes bases de datos estadísti- cas [base de datos en Internet]. San José, Costa Rica: Universidad de Costa Rica. c1996. Hallado en: http://censos.ccp.ucr.ac.cr. Acceso el 8 de octubre de 2004.

7. Organización Panamericana de la Salud. Estadísticas de salud de las Américas: mortalidad, estimaciones y proyecciones demográficas. Washington, D.C.: OPS; 1995. (Publicación científica 556).

8. Organización Panamericana de la Salud. Manual de la clasificación estadística internacional de enfermedades, traumatismos y causas de defunción. 8. ${ }^{a}$ revisión. Washington, D.C.: OPS; 1968.

9. Organización Panamericana de la Salud. Manual de la clasificación estadística internacional de enfermedades, traumatismos y causas de defunción. 9. ${ }^{a}$ revisión. Washington, D.C.: OPS; 1978.

10. Organización Panamericana de la Salud. Clasificación estadística internacional de enfermedades y problemas relacionados con la salud. 10. ${ }^{\text {a }}$ revisión. Washington, D.C.: OPS; 1998.

11. Rosero Bixby L. Tendencias en la mortalidad y esperanza de vida. Costa Rica, 1970-2001.
San José, Costa Rica: Proyecto Estado de la Nación; 2004 (En preparación).

12. Rothman K. Estandarización de tasas. En: Rothman K. Epidemiología moderna. Madrid: Díaz de Santos; 1987.

13. Organización Panamericana de la Salud. Las condiciones de salud de las Américas. Washington, D.C.: OPS; 1995.

14. Murray C, López A. Mortalidad según la causa en ocho regiones del mundo: Global Burden of Disease Study. Lancet (ed. en español). 1997;31:153-61.

15. Levi F, Luchinini F, Negri E, La Vecchia $C$. Trends in mortality from cardiovascular and cerebrovascular diseases in Europe and other areas of the world. Heart. 2002;88:119-24.

16. Alonso I, Puchades M. Evolución de la mortalidad por enfermedades cardiovasculares en la provincia de Toledo de 1975 hasta 1998. Rev Esp Salud Publica. 1995;73(3):365-73.

17. Brotons $C$. Factores de riesgo de la enfermedad coronaria. Atención Primaria. 1990;7: 54-60.

18. Marrugat J, Elousa R, Martí H. Epidemiología de la cardiopatía isquémica en España: estimación del número de casos y las tendencias 
entre 1997 y 2005. Rev Esp Cardiol. 2002;55: $337-46$.

19. Costa Rica, Ministerio de Salud. Memoria institucional, 2003. San José, Costa Rica: Ministerio de Salud; 2003.

20. World Health Organization. WHO Statistical Information System (WHOSIS). Geneva: WHO. Hallado en: http//www.who.int/ whosis. Acceso el 1 de octubre de 2004

21. Nikiforov S, Mamaev V. The development of sex differences in cardiovascular disease mortality: a historical perspective. Am J Public Health. 1998;88:1348-53.

22. Barret-Connor E. Sex differences in coronary health disease. Why are women so superior? Circulation. 1997;95:252-64.

23. Guallar P, Rodríguez F, Banegas J, Lafuente P, Del Rey J. La distribución geográfica de la razón varón/mujer de la mortalidad cardiovascular en España. Gaceta Sanitaria. 2001; 15(4):296-302.

24. Diez A, Merkin S, Aenett D, Chambless L, Massing M, Nieto F, et al. Neighborhood of residence and incidence of coronary heart disease. N Engl J Med. 2001;345:99-106.

25. Moreno C, Turumbay J, García V, Espeleta I, De los Arcos E, Manrique A, et al. Manejo del infarto de miocardio en España. Diferencias interregionales en la actualidad según el registro IBERICA. Rev Esp Cardiol. 2001;54: 419-21.

26. Instituto sobre Alcoholismo y Fármacodependencia. Consumo de drogas en Costa Rica: resultado de la Encuesta Nacional de 1995. San José, Costa Rica: IAFA; 1996.

27. Costa Rica, Instituto sobre Alcoholismo y Farmacodependencia. Compilación de algunos indicadores de medidas alcohólicas y drogas en Costa Rica, 1996. San José, Costa Rica: IAFA; 1998.
28. Ascensio-Rivera M, Rodríguez-Aguilar S, Murrillo-González S. Tabaco y alcohol. En: Costa Rica, Ministerio de Salud. Encuesta basal de factores de riesgo para enfermedades no transmisibles. Cartago 2001. 1. ${ }^{a}$ ed. San José, Costa Rica: Ministerio de Salud; 2003. (Serie de documentos técnicos $\mathrm{N}^{\circ}$ 2).

29. Costa Rica, Ministerio de Salud. Encuesta Nacional de Nutrición. Fascículo 1: Antropometría. San José, Costa Rica: Ministerio de Salud; 1996

30. Ascensio-Rivera M, Rodríguez-Aguilar S, Murrillo-González S. Factores alimentarionutricionales. En: Costa Rica, Ministerio de Salud. Encuesta basal de factores de riesgo para enfermedades no transmisibles. Cartago 2001. 1. ${ }^{\text {a }}$ ed. San José, Costa Rica: Ministerio de Salud; 2003. (Serie de documentos técnicos $\mathrm{N}^{\mathrm{o}} 1$ ).

31. Wessel T, Arant C, Olson M, Johnson D, Reis $\mathrm{S}$, Sharif B, et al. Relationship of physical fitness vs body mass index with coronary artery disease and cardiovascular events in women. JAMA. 2000;292:1179-87.

32. Rodríguez N, Aráuz A, Meza N, Roselló M. Factores aterogénicos de la población costarricense, 1991. Arch Lat Nutr. 1996;46:27-32.

33. Costa Rica, Ministerio de Salud. Encuesta Nacional de Nutrición. Fascículo: Alimentación. San José, Costa Rica: Ministerio de Salud; 1996.

34. Muñoz L, Murillo S, eds. Guías de alimentación: lineamientos metodológicos y criterios técnicos. San José, Costa Rica: Instituto de Nutrición de Centroamérica y Panamá, Universidad de Costa Rica, Escuela de Nutrición; 1995.

35. Guzmán S, Roselló M. Factores socioculturales asociados al consumo de fruta en una población costarricense. Rev Cost Salud Publica. 2003;23:31-7.
36. Expert Panel on Detection, Evaluation and Treatment of High Blood Cholesterol in Adults. Executive summary of the third report of the national cholesterol education program (NCEP) expert panel on detection, evaluation and treatment of high blood cholesterol in adults (Adults Treatment Panel III). JAMA. 2000;285:2486-97.

37. Roselló M, Vargas M, Jiménez JG. Evaluación de factores de riesgo cardiovascular en una población costarricense adulta. Rev Cost Cienc Med. 1996;17:23-33.

38. Morice AC. Análisis de la situación de las enfermedades crónicas no transmisibles en Costa Rica. Tres Ríos, Costa Rica: INCIENSA; 1998.

39. Morice AC, Roselló M, Aráuz AG, Sánchez G, Padilla G. Diabetes mellitus en Costa Rica: un análisis interdisciplinario. Tres Ríos, Costa Rica: INCIENSA; 1999.

40. Puska P, Tuomilehto J, Nissinen A, Vartiainen E, eds. Risk factors and health behavior. En: The North Karelia Project: 20 year results and experiences. Helsinki: University Printing House; 1995. Pp. 77-141.

41. Villar Álvarez F, Banegas Banegas JR. Reducir las enfermedades cardiovasculares. En: Álvarez Dardet C, Peiró S, eds. La salud pública ante los desafíos de un nuevo siglo. Informe SESPAS. Madrid: Sespas; 2000.

42. Siegrist J. Social differentials in chronic disease: what can sociological knowledge offer to explain and possibly reduce them? Soc Sci Med. 1995;41:1603-5.

Manuscrito recibido el 3 de septiembre de 2003. Aceptado para publicación, tras revisión, el 28 de septiembre de 2004
ABSTRACT

Trends in mortality from ischemic heart disease and acute myocardial infarction in Costa Rica, 1970-2001
Objective. To describe epidemiologic trends in mortality from cardiovascular diseases (CVD), ischemic heart disease (IHD), and acute myocardial infarction (AMI) in Costa Rica, by sex and geographic region, between 1970 and 2001.

Methods. We performed a descriptive study of mortality from CVD, IHD, and AMI in Costa Rica between 1970 and 2001. Information was obtained from the Central American Population Center's database. Mortality data for IHD and AMI between 1970 and 2001 were analyzed in accordance with the latest revision of the International Classification of Diseases (ICD). Costa Rica's territory was divided into the following regions: the metropolitan area (8 cantons), the semi-urban area of Valle Central (18 cantons), the rural area of Valle Central (17 cantons), the semi-urban lowlands (12 cantons), and the rural lowlands (26 cantons). Mortality trends by quinquennia (between 1970 and 1999) and for the 2000-2001 biennium were examined in the form of crude mortality rates per 100000 inhabitants for each cause or group of causes, by age, sex, and year of death. All rates were adjusted for sex, age, year of death, and geographic region through the direct method of standardization, using the population of Latin America in 1960 as the standard population.

Results. Mortality from CVD dropped by an average of 33\% (46.6\% among women and $20.2 \%$ among men), while mortality from IHD rose by an average of $18.4 \%(6.1 \%$ among women and $28.4 \%$ among men). The adjusted mortality rate for AMI among men rose by $12.8 \%$ over the study period and dropped slightly by $4.4 \%$ among women. Mortality from CVD, IHD, and AMI was greater in men than in women during the entire study period. Mortality rates for IHD and AMI rose in semi-urban and urban areas, especially in the rural lowlands, where they increased with respect to the $1995-1999$ rates by $123.9 \%$ and $76.9 \%$, respectively.

Conclusions. A reduction in mortality from CVD was noted. The largest rates were seen among men and in persons 75 years of age or older. The largest increases in mortality rates from IHD and AMI were seen in semi-urban and rural areas, among men, and in persons 75 years of age or older. Special attention should be paid to risk factors for CVD, such as smoking, arterial hypertension, overweight and obesity, sedentary habits, and an unhealthy diet. 